\title{
On the Relation between the $S$-matrix and the Spectrum of the Interior Laplacian
}

by

\author{
A. G. RAMM \\ Presented by Bogdan BOJARSKI
}

Summary. The main results of this paper are:

1) a proof that a necessary condition for 1 to be an eigenvalue of the $S$-matrix is real analyticity of the boundary of the obstacle,

2 ) a short proof that if 1 is an eigenvalue of the $S$-matrix, then $k^{2}$ is an eigenvalue of the Laplacian of the interior problem, and that in this case there exists a solution to the interior Dirichlet problem for the Laplacian, which admits an analytic continuation to the whole space $\mathbb{R}^{3}$ as an entire function.

1. Introduction and statement of the result. We consider the obstacle scattering problem in $\mathbb{R}^{3}$, but the argument and the results remain valid in $\mathbb{R}^{n}, n \geq 2$.

Let the obstacle $D \subset \mathbb{R}^{3}$ be a bounded domain with a Lipschitz boundary $S$. Denote by $D^{\prime}=\mathbb{R}^{3} \backslash D$ the exterior domain and by $N$, the unit normal to $S$, pointing into $D^{\prime}$. Let $k>0$ be the wave number, and $S^{2}$ be the unit sphere in $\mathbb{R}^{3}$. The scattering matrix

$$
\mathcal{S}=\mathcal{S}(k)=I-\frac{k}{2 \pi i} A
$$

for the obstacle scattering problem is a unitary operator in $L^{2}\left(S^{2}\right), I$ is the identity operator and $A$ is an integral operator in $L^{2}\left(S^{2}\right)$, whose kernel $A(\beta, \alpha, k)$ is the scattering amplitude, which is defined in formula (5) below. The operator $\mathcal{S}$ has an eigenvalue 1 if and only if equation $A w=0$ has a non-trivial solution. The eigenvalues of $\mathcal{S}$ have 1 as an accumulation point, they all have absolute values equal to 1 since $\mathcal{S}$ is unitary.

2000 Mathematics Subject Classification: 78A45, 35J25.

Key words and phrases: wave scattering by obstacles, $S$-matrix, discrete spectrum, scattering amplitude. 
The following conjecture (the Doron-Smilansky (DS) conjecture) is known:

DS Conjecture. A number $k^{2}>0$ is a Dirichlet eigenvalue of the Laplacian in a bounded domain $D$ if and only if the corresponding $\mathcal{S}$-matrix for the scattering problem by the obstacle $D$ has an eigenvalue 1.

This conjecture is discussed in [1]-[3], and in [3] a counterexample to this conjecture is mentioned.

From the definition of the $\mathcal{S}$-matrix it follows that 1 is its eigenvalue if and only if 0 is an eigenvalue of $A$, that is, equation (12) (see below) has a non-trivial solution.

We prove (see Theorem 2) that if equation (12) has a non-trivial solution, then the boundary $S$ of $D$ is an analytic set. Since generically $S$ is not an analytic set, it follows that the DS conjecture is incorrect. Our result gives a necessary condition for 1 to be an eigenvalue of the $\mathcal{S}$-matrix. This condition is not sufficient (and therefore not sufficient for the DS conjecture to hold for the domain $D$ ).

In [3] it is proved that if $D \subset \mathbb{R}^{2}$ is a bounded domain with a sufficiently smooth boundary $S$, and if 1 is a Dirichlet eigenvalue of $\mathcal{S}$, then $k^{2}$ is a Dirichlet eigenvalue of the Laplacian in $D$. An open problem, stated in [3], is to prove such a statement for $D \subset \mathbb{R}^{n}$ with $n>2$. This is done in our paper by a method different from the one in [3]. Our proof is short and simple.

Let $S_{j}^{2}, j=1,2$, be arbitrary small fixed open subsets of $S^{2}$, and let the boundary conditions on $S$ be either the Dirichlet, Neumann, or Robin conditions.

The following theorem is proved in [5, p. 85]:

Theorem (Ramm). The knowledge of $A(\beta, \alpha, k)$ for all $\alpha \in S_{1}^{2}$ and $\beta \in S_{2}^{2}$, and for a fixed $k>0$, determines $S$ and the boundary conditions on $S$ uniquely.

It follows that the knowledge of the $S$-matrix $\mathcal{S}(k)$ at a fixed $k>0$ determines the boundary $S$ of the obstacle and the boundary condition on $S$ uniquely.

Therefore, the discrete spectrum of the Laplacian in $D$, corresponding to this boundary condition, is uniquely determined by the knowledge of $\mathcal{S}(k)$ at a fixed $k>0$.

This conclusion establishes a relation between the $S$-matrix and the spectrum of the Laplacian in $D$.

Let us now formulate the obstacle scattering problem, introduce basic notions, and formulate our results. 
The scattering solution $u(x, \alpha, k)$ is the solution to the following scattering problem:

$$
\begin{aligned}
& L u:=\left(\nabla^{2}+k^{2}\right) u=0 \quad \text { in } D^{\prime}, \\
& \left.u\right|_{S}=0, \\
& u=u_{0}+v, \quad u_{0}:=e^{i k \alpha \cdot x}, \\
& \frac{\partial v}{\partial r}-i k r=o\left(\frac{1}{r}\right), \quad r:=|x| \rightarrow \infty .
\end{aligned}
$$

Here $\alpha \in S^{2}$ is the incident direction, i.e., the direction of the incident plane wave $u_{0}$, and $v$ is the scattered field which satisfies the radiation condition (4). This condition implies that

$$
v:=v(x, \alpha, k)=A(\beta, \alpha, k) \frac{e^{i k r}}{r}+o\left(\frac{1}{r}\right), \quad r:=|x| \rightarrow \infty, \quad \beta:=\frac{x}{r} .
$$

The function $A:=A(\beta, \alpha, k)$ is called the scattering amplitude. Let us denote by $A: L^{2}\left(S^{2}\right) \rightarrow L^{2}\left(S^{2}\right)$ the operator

$$
A w:=\int_{S^{2}} A(\beta, \alpha, k) w(\alpha) d \alpha .
$$

It is well known (see [5]) that problem (1)-(4) has a unique solution $u(x, \alpha, k)$,

$$
A(\beta, \alpha, k)=-\frac{1}{4 \pi} \int_{S} e^{-i k \beta \cdot s} u_{N}(s, \alpha, k) d s,
$$

where $u_{N}(s, \alpha, k)$ is the normal derivative of the scattering solution $u(x, \alpha, k)$ on $S$, and the following relation holds:

$$
u(x, \alpha, k)=e^{i k \alpha \cdot x}-\int_{S} g(x, s, k) u_{N}(s, \alpha, k) d s .
$$

Here $G$, the resolvent kernel of the Dirichlet Laplacian in the exterior domain $D^{\prime}$, satisfies the following equation:

$$
G(x, y, k)=g(x, y, k)-\int_{S} g(x, s, k) G_{N}(s, y, k) d s,
$$

where

$$
g(x, y, k):=\frac{e^{i k|x-y|}}{4 \pi|x-y|} .
$$

The function $G$ solves the boundary value problem

$$
L G=-\delta(x-y) \quad \text { in } D^{\prime},\left.\quad G\right|_{S}=0,
$$

and satisfies the radiation condition (4).

Let $\sigma$ denote the set of eigenvalues of the Dirichlet Laplacian in $D$. This set is discrete.

It is proved in $[5$, pp. $52-57]$ that: 
(a) The function $A(\beta, \alpha, k)$ admits a meromorphic continuation as a function of $k$ from the ray $(0, \infty)$ to the whole complex $k$-plane.

(b) The scattering amplitude $A(\beta, \alpha, k)$ is analytic in the region $\operatorname{Im} k \geq 0$ (if $D \subset \mathbb{R}^{2 n}$ then $k=0$ is a logarithmic branch point).

(c) $A(\beta, \alpha, k)$ has infinitely many poles on the imaginary axis in the region $\operatorname{Im} k<0$.

(d) As a function of $\alpha$ and $\beta$, the scattering amplitude $A(\beta, \alpha, k)$ admits analytic continuation from $S^{2} \times S^{2}$ to the set $M \times M$, where $M:=$ $\left\{\Theta \in \mathbb{C}^{3}: \Theta \cdot \Theta=1\right\}$, where $\Theta \cdot \omega:=\sum_{j=1}^{3} \Theta_{j} \omega_{j}$. The set $M$ is a non-compact algebraic variety in $\mathbb{C}^{3}$.

Let us now state our basic results:

THEOREM 1. If $\mathcal{S}(k)$ has an eigenvalue 1 , that is, the equation

$$
A w=\int_{S^{2}} A(\beta, \alpha, k) w(\alpha) d \alpha=0
$$

has a non-trivial solution $w$, then $k^{2} \in \sigma$, and there is a solution to the problem $\left(\nabla^{2}+k^{2}\right) W=0$ in $D,\left.W\right|_{S}=0$, which can be extended from $D$ to $\mathbb{R}^{3}$ as a bounded entire function of $x$.

THEOREM 2. If equation (12) has a non-trivial solution, then the boundary $S$ is an analytic set.

An analytic set is a set of zeros of (a finite collection of) analytic functions. One can find the definition and properties of analytic sets in [4, Section 1.4]). If $S$ is an analytic set, then $S$ is a piecewise real analytic surface. Since generically $S$ is not piecewise real analytic surface, it follows from Theorem 2 that the DS conjecture is incorrect.

In Section 2 Theorems 1 and 2 are proved. In the proofs, the following result of the author is used:

Lemma 1 ([5, p. 46]). One has

$$
G(x, y, k)=\frac{e^{i k|y|}}{4 \pi|y|} u(x, \alpha, k)[1+o(1)], \quad|y| \rightarrow \infty, \quad \frac{y}{|y|}=-\alpha,
$$

where $u(x, \alpha, k)$ is the scattering solution, i.e., the solution to (1)-(4).

Lemma 1 yields formula (8) as a consequence of (9), while formula (9) is obtained by Green's formula. Formula (7) follows from (8).

\section{Proofs}

Proof of Theorem 1. Let us prove that if $w \not \equiv 0$ solves (12) then $k^{2} \in \sigma$. Assume that equation (12) has a non-trivial solution $w$. Multiply (7) by 
$w=w(\alpha)$ and integrate over $S^{2}$ with respect to $\alpha$. The result is

$$
\int_{S} e^{-i k \beta \cdot s} p(s) d s=0, \quad p(s):=\int_{S^{2}} u_{N}(s, \alpha, k) w(\alpha) d \alpha .
$$

Let us prove that $p(s) \not \equiv 0$. Indeed, if

$$
p(s)=\int_{S^{2}} u_{N}(s, \alpha, k) w(\alpha) d \alpha=0 \quad \forall s \in S,
$$

then $w(\alpha) \equiv 0$ because the set $\left\{u_{N}(s, \alpha, k)\right\}_{\alpha \in S^{2}}$ is total (dense) in $L^{2}(S)$ for any fixed $k>0$ ([5, p. 162]).

Now equation (14) and Lemma 1 imply that

$$
\nu(x):=\int_{S} \frac{e^{i k|x-s|}}{4 \pi|x-s|} p(s) d s
$$

is identically zero in $D^{\prime}$. Indeed, this $\nu$ solves equation (1), satisfies the radiation condition (4), and (14) implies

$$
\nu(x)=o(1 /|x|), \quad|x| \rightarrow \infty .
$$

Relation (17) and Lemma 1 in [5, p. 25] imply that

$$
\nu(x)=0 \quad \text { in } D^{\prime} .
$$

Therefore, by the jump formula for the normal derivative of the single layer potential $(16)([5$, p. 14] $)$, one gets

$$
\frac{\partial \nu}{\partial N_{+}}=p(s) \not \equiv 0,
$$

where $\partial / \partial N_{+}$denotes the limiting value on $S$ of the normal derivative from inside of $D$.

This implies that $k^{2} \in \sigma$. Indeed, $\nu(x)$ solves the equation

$$
\left(\nabla^{2}+k^{2}\right) \nu=0 \quad \text { in } D^{\prime},
$$

and satisfies the boundary condition

$$
\left.\nu\right|_{S}=0,
$$

due to (18) and the continuity of $\nu$ across $S$. Finally, $\nu \not \equiv 0$ in $D$ because of (19).

Now we prove the existence of a solution to problem (20)-(21) which can be analytically continued to the whole space $\mathbb{R}^{3}$ as an entire function of $x$. 
The reciprocity relation $A(\beta, \alpha, k)=A(-\alpha,-\beta, k)$ (see [5, p. 53] and equation (12) imply

$$
\begin{aligned}
0 & =\int_{S^{2}} A(\beta, \alpha, k) w(\alpha) d \alpha \\
& =-\frac{1}{4 \pi} \int_{S}\left(\int_{S^{2}} e^{i k \alpha \cdot s} w(\alpha) d \alpha\right) u_{N}(s,-\beta) d s \quad \forall \beta \in S^{2} .
\end{aligned}
$$

Since the set $\left\{u_{N}(s, \alpha, k)\right\}_{\alpha \in S^{2}}$ is total (dense) in $L^{2}(S)$ for any fixed $k>0$ $([5$, p. 162]), relation (22) implies

$$
\int_{S^{2}} e^{i k \alpha \cdot s} w(\alpha) d \alpha=0 \quad \forall s \in S .
$$

Therefore, the function

$$
W(x):=\int_{S^{2}} e^{i k \alpha \cdot x} w(\alpha) d \alpha, \quad x \in \mathbb{R}^{3},
$$

satisfies all the requirements mentioned in the last statement of Theorem 1.

Thus, Theorem 1 is proved.

REMARK 1. A similar argument yields the following result:

Let $\sigma_{\mathcal{N}}$ be the set of eigenvalues of the Neumann Laplacian, and $A_{\mathcal{N}}(\beta, \alpha, k)$ the scattering amplitude, corresponding to the plane wave scattering by the obstacle $D$ on the boundary of which the Neumann boundary condition holds. If equation (12), with $A_{\mathcal{N}}$ in place of $A$, has a non-trivial solution, then $k^{2} \in \sigma_{\mathcal{N}}$.

REMARK 2. If $k^{2} \in \sigma$, then any non-trivial solution to (20)-(21) can be written in the form (16) with $p(s)$ defined in (19), and the boundary condition (18) holds. Taking $|x| \rightarrow \infty, x /|x|=\beta$, in (16) and using (18), one obtains

$$
\int_{S} e^{-i k \beta \cdot s} p(s) d s=0 \quad \forall \beta \in S^{2}, \quad p(s) \not \equiv 0 .
$$

Thus, if $k^{2} \in \sigma$, then equation (25) has a non-trivial solution $p(s)$.

Proof of Theorem 2. Suppose equation (12) has a solution $\eta \in L^{2}\left(S^{2}\right)$, $\eta \not \equiv 0$. Then

$$
\int_{S} d s u_{N}(s, \alpha) \int_{S^{2}} e^{-i k \beta \cdot s} \eta(\beta) d \beta=0 \quad \forall \alpha \in S^{2} .
$$

Since the set $\left\{u_{N}(s, \alpha)\right\}_{\alpha \in S^{2}}$ is total in $L^{2}(S)$, one concludes from (26) that

$$
\psi(s)=0 \quad \forall s \in S,
$$


where

$$
\psi(x):=\int_{S^{2}} e^{-i k \beta \cdot x} \eta(\beta) d \beta .
$$

The function $\psi(x)$ is an entire function of $x$, that is, an analytic function of $x \in \mathbb{C}^{3}$. It vanishes on $S$, so $S$ is an analytic set. Generically, the boundary $S$ is not an analytic set.

Thus, Theorem 2 is proved.

REMARK 3. If one uses the reciprocity relation $A(\beta, \alpha, k)=A(-\alpha,-\beta, k)$, then one concludes that zero is an eigenvalue of $A$ if either

$$
\int_{S} e^{-i k \beta \cdot s} \int_{S^{2}} u_{N}(s, \alpha, k) w(\alpha) d \alpha=0 \quad \forall \beta \in S^{2}, \quad w \not \equiv 0,
$$

or

$$
\int_{S}\left(\int_{S^{2}} e^{i k \alpha \cdot s} w(\alpha) d \alpha\right) u_{N}(s,-\beta) d s=0 \quad \forall \beta \in S^{2}, \quad w \not \equiv 0 .
$$

The last relation implies equation (28) (with $\beta=-\alpha$ and $\eta(\beta)=w(\alpha)$ ).

Set $T_{k} p:=\int_{S} g(s, t, k) p(t) d t$ and $U:=U(x, k):=\int_{S} g(x, t, k) p(t) d t$, so $\left.U\right|_{S}=T_{k} p$.

REMARK 4. The operator $T_{k}^{-1}$ has simple poles at the points $k^{2}=k_{j}^{2}$, where $k_{j}^{2} \in \sigma$.

Remark 4 shows that the knowledge of the set of poles of the operator $T_{k}^{-1}$ allows one to find the spectrum of the interior Dirichlet Laplacian in $D$.

Proof of Remark 4. Consider the equation $T_{k} p=f$. Then

$$
U(x)=\int_{S} g(x, t, k) p(t) d t
$$

solves the problem

$$
\left(\nabla^{2}+k^{2}\right) U=0 \quad \text { in } D,\left.\quad U\right|_{S}=f .
$$

Let

$$
\left(\nabla^{2}+k^{2}\right) \Gamma=-\delta(x-y) \quad \text { in } D,\left.\quad \Gamma\right|_{S}=0 .
$$

Then Green's formula yields the following representation of the solution to problem (30):

$$
U(x)=-\int_{S} f(t) \Gamma_{N_{t}}(t, x, k) d t, \quad x \in D, \quad k^{2} \neq k_{j}^{2} .
$$

Since $\Gamma(x, y, k)=\sum_{j=1}^{\infty} \frac{\phi_{j}(x) \overline{\phi_{j}(y)}}{k^{2}-k_{j}^{2}}$ has a simple pole at $k^{2}=k_{j}^{2}$, the claim is proved. Here $\phi_{j}$ are the normalized eigenfunctions of the Dirichlet Laplacian in $D$. 


\section{References}

[1] B. Dietz, J.-P. Eckmann, C.-A. Pillet and U. Smilansky, Inside-outside duality for planar billiards: A numerical study, Phys. Rev. E 51 (1995), 4222-4231.

[2] E. Doron and U. Smilansky, Semiclassical quantization of chaotic billiards: a scattering approach, Nonlinearity 5 (1992), 1055-1084.

[3] J.-P. Eckmann and C.-A. Pillet, Spectral duality for planar billiards, Comm. Math. Phys. 170 (1995), 283-313.

[4] B. A. Fuks, Theory of Analytic Functions of Several Variables, Amer. Math. Soc., Providence, RI, 1963.

[5] A. G. Ramm, Scattering by Obstacles, Reidel, Dordrecht, 1986.

A. G. Ramm

Mathematics Department

Kansas State University

Manhattan, KS 66506-2602, U.S.A.

E-mail: ramm@math.ksu.edu

Received April 9, 2009;

received in final form June 14, 2009 